\title{
Asymptotic Capacity of Multi-Level Amplify-and-Forward Relay Networks
}

\author{
Shu-ping Yeh \\ Department of Electrical Engineering \\ Stanford University \\ Stanford, CA 94305-9510, USA \\ shuping@stanford.edu
}

\author{
Olivier Lévêque \\ Faculté Informatique et Communications \\ Ecole Polytechnique Fédérale de Lausanne \\ 1015 Lausanne, Switzerland \\ olivier.leveque@epfl.ch
}

\begin{abstract}
This paper analyzes the capacity of a wireless relay network composed of a large number of nodes that operate in an amplify-and-forward mode and that divide into a fixed number of levels. The capacity computation relies on the study of products of large random matrices, whose limiting eigenvalue distribution is computed via a set of recursive equations.
\end{abstract}

\section{INTRODUCTION}

A typical relay network consists of three groups of nodes: the sources, the destinations and the relays. In one-direction transmission, the messages are sent from the sources to the destinations with assistance from the relays. A special case is when there is no direct link between the information sources and the destinations. Direct links are absent when the sources and the destinations are far from each other or when there are obstructions between the sources and the destinations. Therefore, the sources must first send the messages to the relays. Then the relays forward the received signals to the destinations. In an amplify-and-forward (AF) relay network, the relays simply scale the received signals according to their power constraint and forward the scaled signals to the destinations. This amplify-and-forward operation is a reasonable strategy when relays have a limited computation power and no centralized control nor feedback exists. The AF relay network is illustrated in Figure 1.

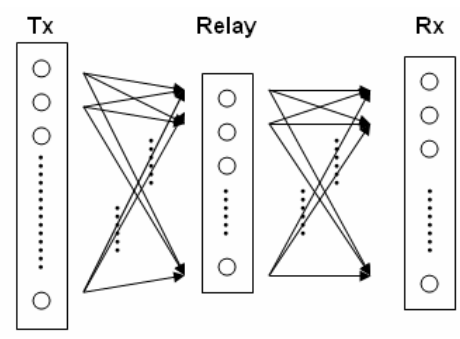

Fig. 1. AF relay network

When the sources and the destinations are too distant or numerous obstructions exist, multiple levels of relays may be needed. In a multi-level AF relay network, the first level of relays amplify the received signals and forward the amplified signals to the next level of relays. The next level of relays repeats the same operation, and so on. Finally, the last level of relays forwards the signals to the destinations. Figure 2 illustrates the multi-level AF relay network.

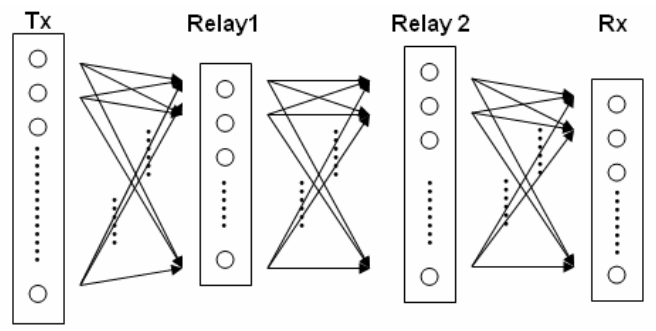

Fig. 2. 2-level AF relay network

The multi-level amplify-and-forward strategy allows saving of both computation time and energy at the relays, at the price of noise amplification at each level. This paper quantifies precisely the loss incured by this noise amplification when the number of nodes gets large, but the number of relay levels remains fixed. The capacity of the multi-level AF scheme is the expectation of the log determinant of a product of random matrices. This work needs classical tools from Random Matrix Theory throughout, as developed in [1]-[4].

The analysis of the capacity-scaling behavior of AF relay networks has also been conducted by Morgenshtern and Bolcskei in [5]-[7]. They use results in [4], but consider only one level of relays. This paper modifies Morgenshtern and Bolcskei's approach to obtain a general formula for multi-level AF relay networks. Multi-level AF networks have also been considered by Borade and Zheng in [8], but in the high SNR regime. Our contribution is a fixed SNR analysis.

\section{SyStem ModeL}

This paper follows the same system model as in [5]-[7]. Under the simplified assumption of a flat-fading channel and perfectly synchronized transmission and reception among all terminals. Suppose there are $m_{T}$ source terminals and $m_{R}$ destination terminals. If the source terminals are far from the destination 
terminals and no direct link between them exists, there will be at least one level of relay terminals present to assist the transmission. Suppose that there are in total $K$ levels of relays. Aversion of interference suggests a time-division transmission strategy: In every transmission cycle, there are $K+1$ time slots. At the first time slot, the source terminals transmit the signals to the first level of relays. Each level of relay terminals performs the AF operation; that is, they amplify their received signals and forward them to the next level of relays. The $k^{t h}$ level of relays transmits the messages to the $(k+1)^{t h}$ level of relays at the $(k+1)^{t h}$ time slot. At the $(K+1)^{t h}$ time slot, the messages arrive at the destination nodes from the last level of relays.

Let $l_{k}$ be the number of relays at level $k$ for $k=1, \ldots, K$ and $l_{0}=m_{T}, l_{K+1}=m_{R}$. Let then $H_{k}=\left\{H_{k}^{i, j}\right\} \in \mathbb{C}^{l_{k} \times l_{k-1}}$ denote the channel gain matrix between the $(k-1)^{t h}$ and $k^{t h}$ level of relays (where $k=0$ corresponds to the source nodes and $k=K+1$ to the destination nodes). The matrices $H_{k}$ are independent and their entries are i.i.d. $\sim \mathcal{N}_{\mathbb{C}}(0,1)$ random variables, varying ergodically over time (fast fading assumption). Additive white Gaussian noise $Z_{k}$ (with unit variance) adds at each level $k . Z=Z_{K+1}$ is thus the noise at the final destinations.

There is no cooperation nor channel-state information (CSI) at the source and relay terminals, but full cooperation and full CSI (i.e. the knowledge of the realizations of all the random matrices $\left.H_{0}, \ldots, H_{K+1}\right)$ is assumed at the destinations. The power constraint at each node in the network is inversely proportional to the number of nodes at its level.

Let $Y_{k}=\left[y_{k 1}, y_{k 2}, \ldots, y_{k l_{k}}\right]$ and $X_{k}=\left[x_{k 1}, x_{k 2}, \ldots, x_{k l_{k}}\right]$ be the signals received and transmitted by the $k^{\text {th }}$ level of relay, respectively. Then the K-level AF relay channel can be modeled as:

$$
\begin{aligned}
Y_{1} & =H_{1} X+Z_{1} \\
Y_{k} & =H_{k} X_{k-1}+Z_{k}, \quad k=2, \ldots, K \\
Y & =H_{K+1} X_{K}+Z
\end{aligned}
$$

For each level of relays, the received signals are scaled according to the power constraint at this level. Let the scaling factor of the $k^{t h}$ level be $\alpha_{k}$. Therefore, the scaling is

$$
X_{k}=\alpha_{k} Y_{k}, \quad k=1, \ldots, K
$$

The total power constraint at the $k^{\text {th }}$ level is $P_{k}$. For the first level,

$P_{1}=\mathbb{E}\left[X_{1}^{*} X_{1}\right]=\alpha_{1}^{2} \mathbb{E}\left[Y_{1}^{*} Y_{1}\right]=\alpha_{1}^{2} \mathbb{E}\left[\left(H_{1} X+Z_{1}\right)^{*}\left(H_{1} X+Z_{1}\right)\right]$

Since $X, H_{1}$ and $Z_{1}$ are independent of each other,

$$
\begin{aligned}
P_{1} & =\alpha_{1}^{2}\left(\mathbb{E}\left[X^{*} \mathbb{E}\left[H_{1}^{*} H_{1}\right] X\right]+\mathbb{E}\left[Z_{1}^{*} Z_{1}\right]\right) \\
& =\alpha_{1}^{2}\left(\mathbb{E}\left[X^{*}\left(l_{1} I\right) X\right]+l_{1}\right)=\alpha_{1}^{2} l_{1}(P+1)
\end{aligned}
$$

Similarly, for other levels:

$$
P_{k}=\mathbb{E}\left[X_{k}^{*} X_{k}\right]=\alpha_{k}^{2} l_{k}\left(P_{k-1}+1\right), \quad k=2, \ldots, K
$$

Define $\beta_{1}=\frac{P+1}{P_{1}} ; \beta_{k}=\frac{P_{k-1}+1}{P_{k}}, k=2,3, \ldots, K$. The scaling factors can then be written as $\alpha_{k}=\frac{1}{\sqrt{\beta_{k} l_{k}}}, k=1, \ldots, K$. For completeness, $\beta_{0}=\frac{1}{P}$ and $\alpha_{0}=\frac{1}{\sqrt{\beta_{0} l_{0}}}=\sqrt{\frac{P}{m_{T}}}$.

A recursive definition of a new series of matrices is $\left\{G_{k}\right\}_{k=0}^{K+1}$, where $G_{K+1}=I$ and

$$
G_{k}=\alpha_{k} G_{k+1} H_{k+1}, \quad k=K, \ldots, 0
$$

Therefore, the overall channel can be expressed via $G_{k}$ as:

$$
\begin{aligned}
Y & =G_{K} Y_{K}+Z=G_{K-1} Y_{K-1}+G_{K} Z_{K}+Z \\
& =\ldots=G_{1} H_{1} X+\sum_{k=1}^{K} G_{k} Z_{k}+Z
\end{aligned}
$$

$G_{1} H_{1} X$ is the signal part and $\sum_{k=1}^{K} G_{k} Z_{k}+Z$ is the noise part. Perfect receiver channel-state information is assumed, so the destination terminals know all the $H_{k}$ 's. For ease of notation, a new series of matrices $\left\{\Sigma_{k}\right\}_{k=1}^{K+1}$ is introduced, where $\Sigma_{K+1}=I$ and

$$
\Sigma_{k}=\mathbb{E}\left(\left(Z+\sum_{i=k}^{K} G_{i} Z_{i}\right)\left(Z+\sum_{i=k}^{K} G_{i} Z_{i}\right)^{*} \mid H_{1}, \ldots, H_{K+1}\right)
$$

for $k=1, \ldots, K$. The covariance matrix of the noise part is then $\Sigma_{1}$. Also, since $Z_{1}, Z_{2}, \ldots Z_{K}$ and $Z$ have i.i.d. $\sim \mathcal{N}_{\mathbb{C}}(0,1)$ components, the matrices $\left\{\Sigma_{k}\right\}_{k=1}^{K}$ obey the following recursive relationship

$$
\Sigma_{k}=\Sigma_{k+1}+G_{k} G_{k}^{*}, \quad k=K, \ldots, 1
$$

Capacity computation requires knowledge of both the noise and the signal covariance matrices. Based on the Gaussian channel assumption, the capacity is achieved when the entries of $X$ are jointly Gaussian. Suppose the covariance matrix of $X$ is $Q$. Since by assumption, there is no CSI at the sources and the entries of $H_{1}$ are i.i.d. Gaussian and independent of $G_{1}$, [9] relates that the optimal $X$ is distributed according to $\mathcal{N}_{\mathbb{C}}\left(0, \frac{P}{m_{T}} I\right)$. Thus, the covariance matrix of the signal part is $\frac{P}{m_{T}} G_{1} H_{1} H_{1}^{*} G_{1}^{*}=G_{0} G_{0}^{*}$.

The overall capacity is then

$$
\begin{aligned}
C & =\frac{1}{K+1} \mathbb{E} \log \operatorname{det}\left(I+\frac{P}{m_{T}} \Sigma_{1}^{-\frac{1}{2}} G_{1} H_{1} H_{1}^{*} G_{1}^{*} \Sigma_{1}^{-\frac{1}{2}}\right) \\
& =\frac{1}{K+1} \mathbb{E} \log \operatorname{det}\left(I+\frac{P}{m_{T}} H_{1}^{*} G_{1}^{*} \Sigma_{1}^{-1} G_{1} H_{1}\right)
\end{aligned}
$$

(the $\frac{1}{K+1}$ term comes from the use of the time-division scheme). We analyze the above capacity when $m_{T}, m_{R}$, and all $l_{k}$ 's tend to infinity, and they also tend to some given ratios while going to infinity, say, $\frac{l_{1}}{m_{T}} \rightarrow c_{1} ; \frac{l_{k}}{l_{k-1}} \rightarrow c_{k}, k=$ $2, \ldots, K ; \frac{m_{R}}{l_{K}} \rightarrow c_{K+1}$.

\section{CAPACITY ANALYSIS}

A powerful tool for analyzing the limiting eigenvalue distribution (LED) of large dimensional random matrices is the Stieltjes transform. A thorough discussion of its applications can be found in [2]. Let $F$ be a distribution on $\mathbb{R}$ (here and in the rest of the paper, one identifies a distribution on $\mathbb{R}$ with its 
cumulative distribution function). Then its Stieltjes transform is defined as

$$
g(z) \equiv \int_{-\infty}^{\infty} \frac{1}{x-z} d F(x), z \in \mathbb{C}^{+} \equiv\{z \in \mathbb{C}: \operatorname{Im}(z)>0\}
$$

with the inversion formula

$$
\lim _{\epsilon \downarrow 0} \int_{x_{1}}^{x_{2}} \frac{1}{\pi} \operatorname{Im}(g(x+i \epsilon)) d x=F\left(x_{2}\right)-F\left(x_{1}\right)
$$

and the useful fact that a sequence of distributions converges to a limit if and only if the corresponding sequence of Stieltjes transforms converges. The following result is a straightforward consequence of a result by Silverstein [4]. Recall that the empirical eigenvalue distribution of an $n \times n$ Hermitian matrix $A_{n}$ with real eigenvalues $\lambda_{1}, \ldots, \lambda_{n}$ is given by $F_{A_{n}}(x)=$ $\frac{1}{n} \sum_{k=1}^{n} 1_{\left\{x \geq \lambda_{k}\right\}}$.

Theorem 1: Let $n, N \geq 1$ and let us assume that:

(a) $X_{n}=\left(X_{i j}^{n}\right)$ is an $n \times N$ random matrix with i.i.d. entries such that $\mathbb{E}\left|X_{11}^{n}-\mathbb{E} X_{11}^{n}\right|^{2}=1$;

(b) $N=N(n)$ with $n / N \rightarrow c>0$ as $n \rightarrow \infty$;

(c) $T_{n}$ is an $n \times n$ random Hermitian non-negative definite matrix such that its empirical eigenvalue distribution $F_{T_{n}}$ converges almost surely, as $n \rightarrow \infty$, to a (deterministic) distribution $F_{T}$, with corresponding Stieltjes transform $g_{T}$;

(d) $X_{n}$ and $T_{n}$ are independent.

Let $A_{n}=\frac{1}{N} X_{n}^{*} T_{n} X_{n}$. Then its empirical eigenvalue distribution $F_{A_{n}}$ converges almost surely, as $n \rightarrow \infty$, to a (deterministic) distribution $F_{A}$, whose Stieltjes transform $g_{A}$ satisfies

$$
z g_{A}(z)+1=c\left(\frac{-1}{g_{A}(z)} g_{T}\left(\frac{-1}{g_{A}(z)}\right)+1\right)
$$

in the sense that, for each $z \in \mathbb{C}^{+}, g=g_{A}(z)$ is the unique solution to (7) such that $g \in \mathbb{C}^{+}$.

In the particular case where $T_{n}=I, F_{T}(x)=I_{\{x \geq 1\}}$ and $g_{T}(z)=1 /(1-z)$, so the above equation reads

$$
z g_{A}(z)+1=\frac{c g_{A}(Z)}{1+g_{A}(z)},
$$

and its solution is the Stieltjes transform of the well-known Marčenko-Pastur distribution [1].

In order to illustrate why Silverstein's theorem can be used to compute the capacity expression (4), the single-level AF relay network will be examined first. The single-level case provides general guidance for computing the general multilevel problem. We then derive a set of recursive equations that can be used to compute the Stieltjes transform of the LED of the multi-level AF relay network.

1) Single-Level AF Relay Network: The single level AF relay network has also been analyzed in [5]-[7]. This paper suggests another perspective on the formulation of the problem. The advantage is extension to the multi-level case.

When $K=1, G_{1}=\alpha_{1} H_{2}$ and $\Sigma_{1}=I+G_{1} G_{1}^{*}$. Let $T_{1}=$ $G_{1}^{*} \Sigma_{1}^{-1} G_{1}, M_{1}=\sqrt{\frac{1}{m_{T}}} T_{1}^{\frac{1}{2}} H_{1}$ and $M_{2}=\sqrt{\frac{1}{l_{1}}} H_{2}$. Then, from (4),

$$
C_{1 \text {-level AF }}=\frac{1}{2} \mathbb{E} \log \operatorname{det}\left(I+P M_{1}^{*} M_{1}\right)
$$

where

$$
\begin{aligned}
M_{1}^{*} M_{1} & =\frac{1}{m_{T}} H_{1}^{*} T_{1} H_{1} \\
T_{1} & =M_{2}^{*}\left(\beta_{1}+M_{2} M_{2}^{*}\right)^{-1} M_{2} \\
M_{2}^{*} M_{2} & =\frac{1}{l_{1}} H_{2}^{*} H_{2}
\end{aligned}
$$

Capacity computation first finds the LED of $M_{1}^{*} M_{1}$. An equation for the Stieltjes transform of the LED of $M_{1}^{*} M_{1}$ is given in the following theorem. From now on, notation $g_{M_{k}^{*} M_{k}}$ abbreviates $g_{k}$.

Theorem 2: For matrices $M_{1}, M_{2}$ and $T_{1}$ satisfying (9), (10) and (11) with $\frac{l_{1}}{m_{T}} \rightarrow c_{1}$ and $\frac{m_{R}}{l_{1}} \rightarrow c_{2}$, the Stieltjes transform $g_{1}$ of the LED of $M_{1}^{*} M_{1}$ satisfies the following equations:

$$
\begin{aligned}
z g_{1}(z) & +1 \\
& =\frac{c_{1} g_{1}(z)}{1+g_{1}(z)}\left(\frac{-\beta_{1}}{1+g_{1}(z)} g_{2}\left(\frac{-\beta_{1}}{1+g_{1}(z)}\right)+1\right) \\
z g_{2}(z) & +1=\frac{c_{2} g_{2}(z)}{1+g_{2}(z)}
\end{aligned}
$$

where $g_{1}: \mathbb{C}^{+} \rightarrow \mathbb{C}^{+}$and $g_{2}: \mathbb{C}^{+} \rightarrow \mathbb{C}^{+}$.

Proof: The basic proof idea goes as follows. From (9), if $T_{1}$ is random Hermitian nonnegative definite and independent of $H_{1}$, with its eigenvalue distribution converging almost surely as $m_{T} \rightarrow \infty$, then Theorem 1 applies to compute the LED of $M_{1}^{*} M_{1}$. On the other hand, (11) implies that the eigenvalue distribution of $M_{2}^{*} M_{2}$ converges a.s., with its Stieltjes transform $g_{2}$ satisfying (13) with $g_{2}: \mathbb{C}^{+} \rightarrow \mathbb{C}^{+}$. Therefore, the missing link is the relationship between the eigenvalues of $T_{1}$ and $M_{2}^{*} M_{2}$. This relationship is given in the following lemma, whose proof is straightforward.

Lemma 1: Consider two matrices $T_{1} \in \mathbb{C}^{l_{1} \times l_{1}}$ and $M_{2} \in$ $\mathbb{C}^{l_{1} \times m_{R}}$ satisfying (10), and denote by $t_{i}$ and $m_{i}$ the eigenvalues of $T_{1}$ and $M_{2}^{*} M_{2}$ respectively, where $t_{1} \leq t_{2} \leq \ldots \leq t_{l_{1}}$ and $m_{1} \leq m_{2} \leq \ldots \leq m_{l_{1}}$. Then

$$
t_{k}=\frac{m_{k}}{\beta_{1}+m_{k}} \quad k=1, \ldots, l_{1}
$$

Therefore, their eigenvalue distributions satisfy

$$
F_{M_{2}^{*} M_{2}}(x)=F_{T_{1}}\left(\frac{x}{\beta_{1}+x}\right)
$$

and their corresponding Stieltjes transforms satisfy

$$
z g_{T_{1}}(z)+1=\frac{1}{1-z}\left(\frac{\beta_{1} z}{1-z} g_{2}\left(\frac{\beta_{1} z}{1-z}\right)+1\right)
$$

Since $M_{2}^{*} M_{2}$ is Hermitian nonnegative definite, $T_{1}$ is also Hermitian nonnegative definite, and its eigenvalue distribution converges a.s. Therefore, Theorem 1 applies to find the Stieltjes transform $g_{1}$ : combining (7) and (16) yields the conclusion that $g_{1}$ satisfies (12) with $g_{1}: \mathbb{C}^{+} \rightarrow \mathbb{C}^{+}$. 
We can find $g_{1}$ by solving the overall $4^{t h}$-order equation combining (12) and (13). Only one of the roots of the $4^{\text {th }}$ order equation satisfies both $g_{1}: \mathbb{C}^{+} \rightarrow \mathbb{C}^{+}$and $g_{2}: \mathbb{C}^{+} \rightarrow$ $\mathbb{C}^{+}$. From $g_{1}$, the inverse Stieltjes transform (6) provides the corresponding LED $F_{1}$. The single-level AF channel capacity is then given by

$$
C_{1 \text {-level } \mathrm{AF}} \sim \frac{m_{T}}{2} \int \log (1+P x) d F_{1}(x)
$$

as $m_{T} \rightarrow \infty$. Therefore, as already noticed in [7], the capacity increases linearly with the number of nodes at each level.

2) Multi-Level AF Relay Network: Generalization of the previous result to the multi-level case requires a recursive transformation of the matrices for iterative application of Lemma 1 and Theorem 1 to compute the Stieltjes transform of the LED of the matrix in (4).

In addition to the two series of matrices $\left\{G_{k}\right\}$ and $\left\{\Sigma_{k}\right\}$ defined in (1) and (3), two new series of matrices are:

$$
\begin{aligned}
T_{k} & =G_{k}^{*} \Sigma_{k}^{-1} G_{k} \\
M_{k} & =\sqrt{\beta_{k-1}} \Sigma_{k}^{-\frac{1}{2}} G_{k-1}, \quad k=1, \ldots, K+1
\end{aligned}
$$

Derivation of a recursive relationship between $\left\{T_{k}\right\}$ and $\left\{M_{k}\right\}$ requires the following matrix-inversion lemma, whose proof is straightforward.

Lemma 2: If $\Sigma$ is positive definite, then

$$
\left(\Sigma+G G^{*}\right)^{-1}=\Sigma^{-\frac{1}{2}}\left(I+\Sigma^{-\frac{1}{2}} G G^{*} \Sigma^{-\frac{1}{2}}\right)^{-1} \Sigma^{-\frac{1}{2}}
$$

Use the above lemma and the recursive formulas (1),(3) for $G_{k}, \Sigma_{k}$, leads to the following lemma.

Lemma 3: Let $T_{K+1}=I$. The recursive relationship between $T_{k}$ and $M_{k}$ can be written as

$$
\begin{gathered}
M_{k}^{*} M_{k}=\frac{1}{l_{k-1}} H_{k}^{*} T_{k} H_{k}, \quad k=1, \ldots, K+1 \\
T_{k}=M_{k+1}^{*}\left(\beta_{k} I+M_{k+1} M_{k+1}^{*}\right)^{-1} M_{k+1}, k=1, \ldots, K
\end{gathered}
$$

Proof: (19) comes directly from the definition of $T_{k}$ and $M_{k}$ and the fact that $G_{k-1}=\alpha_{k-1} G_{k} H_{k}$ :

$$
\begin{aligned}
M_{k}^{*} M_{k} & =\beta_{k-1} G_{k-1}^{*} \Sigma_{k}^{-1} G_{k-1} \\
& =\beta_{k-1} \alpha_{k-1}^{2} H_{k}^{*} G_{k}^{*} \Sigma_{k}^{-1} G_{k} H_{k}=\frac{1}{l_{k-1}} H_{k}^{*} T_{k} H_{k}
\end{aligned}
$$

The derivation of (20) involves the recursion $\Sigma_{k}=\Sigma_{k+1}+$ $G_{k} G_{k}^{*}$ and Lemma 2:

$$
\begin{aligned}
T_{k} & =G_{k}^{*} \Sigma_{k}^{-1} G_{k}=G_{k}^{*}\left(\Sigma_{k+1}+G_{k} G_{k}^{*}\right)^{-1} G_{k} \\
& =G_{k}^{*} \Sigma_{k+1}^{-\frac{1}{2}}\left(I+\Sigma_{k+1}^{-\frac{1}{2}} G_{k} G_{k}^{*} \Sigma_{k+1}^{-\frac{1}{2}}\right)^{-1} \Sigma_{k+1}^{-\frac{1}{2}} G_{k} \\
& =M_{k+1}^{*}\left(\beta_{k} I+M_{k+1} M_{k+1}^{*}\right)^{-1} M_{k+1}
\end{aligned}
$$

Again, capacity computation is through the LED of $M_{1}^{*} M_{1}=$ $\frac{1}{l_{0}} H_{1}^{*} T_{1} H_{1}=\frac{1}{m_{T}} H_{1}^{*} G_{1}^{*} \Sigma_{1}^{-1} G_{1} H_{1}$. The similarity between (9), (10) and (19), (20) suggests a recursive way to compute the Stieltjes transform of the LED of $M_{1}^{*} M_{1}$ for the multilevel case.

Theorem 3: For matrix series $\left\{M_{k}\right\}_{k=1}^{K+1}$ and $\left\{T_{k}\right\}_{k=1}^{K+1}$ satisfying (19) and (20), the Stieltjes transforms $g_{k}$ of the LED of $M_{k}^{*} M_{k}$ satisfy the following equations for $k=1, \ldots, K$ :

$$
\begin{aligned}
& z g_{k}(z)+1 \\
& \quad=\frac{c_{k} g_{k}(z)}{1+g_{k}(z)}\left(\frac{-\beta_{k}}{1+g_{k}(z)} g_{k+1}\left(\frac{-\beta_{k}}{1+g_{k}(z)}\right)+1\right) \\
& z g_{K+1}(z)+1=\frac{c_{K+1} g_{K+1}(z)}{1+g_{K+1}(z)}
\end{aligned}
$$

where $g_{k}: \mathbb{C}^{+} \rightarrow \mathbb{C}^{+}, k=1, \ldots, K+1$.

Proof: (20) implies that $T_{k}$ is Hermitian nonnegative definite. Lemma 1 provides the relationship between the eigenvalues of $M_{k+1}^{*} M_{k+1}$ and $T_{k}$. As long as the eigenvalues distribution of $M_{k+1}^{*} M_{k+1}$ converges a.s., then the eigenvalue distribution of $T_{k}$ converges a.s.

From Theorem 1, the eigenvalue distribution of $M_{k}^{*} M_{k}$ converges a.s. if the eigenvalue distribution of $T_{k}$ converges a.s. Since $T_{K+1}=I$, the convergence of the eigenvalue distribution of $M_{K+1}^{*} M_{K+1}$ is ensured, and thus, the eigenvalue distribution of $T_{K}$ also converges almost surely. This again ensures the convergence of the eigenvalue distribution of $M_{K}^{*} M_{K}$ and $T_{K-1}$, and so on. By induction, we conclude that the eigenvalue distribution of $M_{k}^{*} M_{k}, k=1, \ldots, K+1$ converges almost surely.

Finally, combining (7), (8) and (16), we obtain the recursive equations (21) and (22) for the Stieltjes transform $g_{k}$ of the LED of $M_{k}^{*} M_{k}, k=1, \ldots K+1$.

Computing the capacity (4) requires the knowledge of the LED of $M_{1}^{*} M_{1}$. In order to be explicit, let us define a new sequence $\left\{z_{k}\right\}_{k=1}^{K+1}$, where $z_{1}=z, z_{k+1}=\frac{-\beta_{k}}{1+g_{k}\left(z_{k}\right)}, k=1, \cdots, K$. We can then rewrite (21) and obtain for $k=1, \ldots, K$ :

$$
\begin{gathered}
g_{k+1}\left(z_{k+1}\right)=\frac{-1}{z_{k+1}}\left(1-\frac{1+g_{k}\left(z_{k}\right)}{c_{k} g_{k}\left(z_{k}\right)}\left(z_{k} g_{k}\left(z_{k}\right)+1\right)\right) \\
=\frac{1+g_{k}\left(z_{k}\right)}{\beta_{k}}\left(1-\frac{1+g_{k}\left(z_{k}\right)}{c_{k} g_{k}\left(z_{k}\right)}\left(z_{k} g_{k}\left(z_{k}\right)+1\right)\right)
\end{gathered}
$$

Above equation suggests expression of $g_{k}\left(z_{k}\right), k=2, \ldots, K+$ 1 , in terms of $z$ and $g_{1}(z)$. Therefore, substitution of the expression for $g_{K+1}\left(z_{K+1}\right)$ in terms of $z$ and $g_{1}(z)$ into (22) yields an equation for $g_{1}(z)$. The overall equation for $g_{1}$ has order $2^{K+1}$, and can be solved numerically. Only one of the roots satisfies $g_{k}\left(z_{k}\right) \in \mathbb{C}^{+}, \forall k=1, \ldots, K+1$. The LED $F_{1}$ of $M_{1}^{*} M_{1}$ is computed by the inverse Stieltjes transform (6), and the capacity is given by the formula

$$
C_{\mathrm{K}-\text { level AF }} \sim \frac{m_{T}}{K+1} \int \log (1+P x) d F_{1}(x)
$$

as $m_{T} \rightarrow \infty$. The capacity increases linearly with the number of nodes at each level. Finally, it is possible to show that when $c_{1} \rightarrow \infty$ and $\prod_{k=1}^{K+1} c_{k}=c$ remains fixed, $F_{1}$ converges to a classical Marčenko-Pastur distribution, whose Stieltjes transform $g_{1}$ is solution of an equation of the type (8). 


\section{NUMERICAl SimUlations}

First, this section compares the theoretical LED that we obtain in Section III-.2 with the empirical eigenvalue distribution of a finite-dimensional matrix. Figure 3 shows that there is indeed a fairly good agreement between the two, even for a small number of nodes in the network.

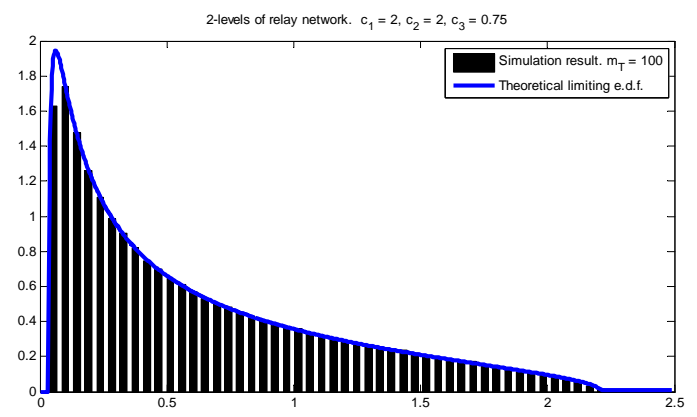

Fig. 3. Limiting versus empirical eigenvalue distribution

Next of interest is the effect of the number of relays upon the LED and the capacity. The ratio between the number of source and destination terminals is fixed, while the number of relays in between varies. When there is only one level of relays, the LED and the capacity are shown in figure 4 and 5 respectively.

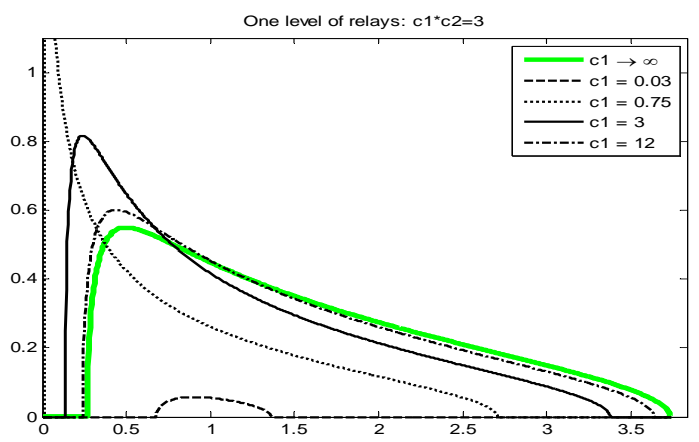

Fig. 4. Varying the number of relays in a 1-level AF relay network: LED

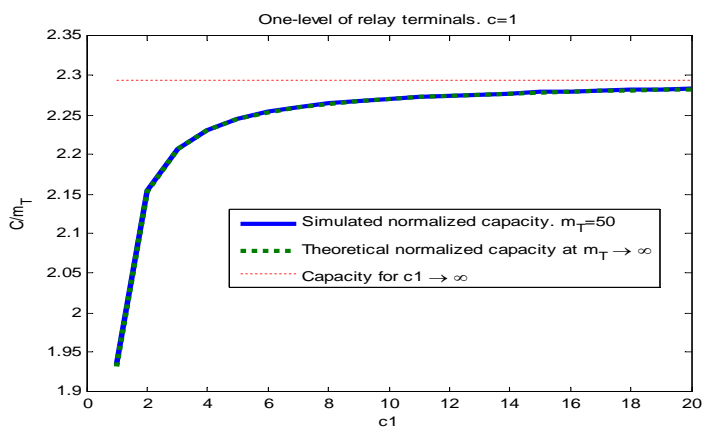

Fig. 5. Varying the number of relays in a 1-level AF relay network: capacity

Figure 6 plots the limiting eigenvalue distribution of a twolevel AF relay channel. We vary the ratio between the number of relays at the first level and the number of source terminals, while the ratio between the numbers of relays in the first and second levels is fixed.

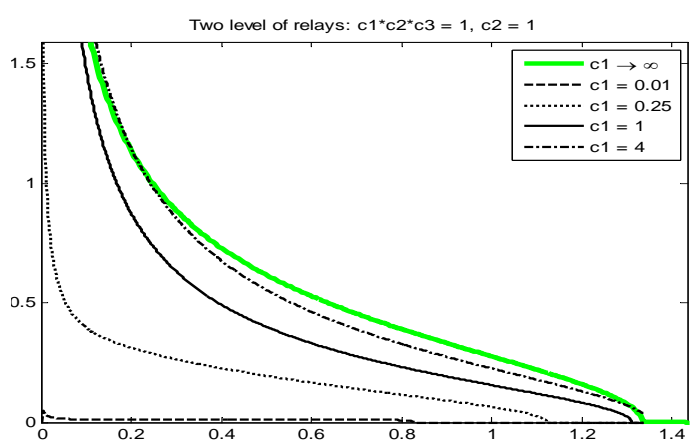

Fig. 6. Varying the number of first-level relays in a 2-level AF relay network

\section{CONCLUSION}

In this paper, the capacity of a large scale multi-level AF relay network has been analyzed. The capacity formula is expressed by means of the limiting eigenvalue distribution of a random matrix, whose Stieltjes transform is shown to satisfy a set of recursive equations. A general procedure for solving explicitly these equations has been proposed, from which one can deduce both the limiting eigenvalue distribution and the capacity.

\section{ACKNOWLEDGEMENT}

We would like to thank Helmut Bölcskei and Veniamin Morgenshtern for suggesting the problem of studying multilevel AF networks and sharing an early version of their paper [6] with us. Part of this work was performed while Olivier Lévêque was at Stanford University, supported by Swiss NSF grant No PA002-108976.

\section{REFERENCES}

[1] V. A. Marčenko and L. A. Pastur, The Distribution of Eigenvalues in Certain Sets of Random Matrices, Math USSR-Sbornik, Vol. 72, No. 4, pp. 507-536, 1967.

[2] Z. D. Bai, Methodologies in Spectral Analysis of Large Dimensional Random Matrices. A Review, Statistica Sinica, Vol. 9, pp. 611-677, 1999.

[3] Z. D. Bai, J. .W. Silverstein, On the Empirical Distribution of Eigenvalues of a Class of Large Dimensional Random Matrices, Journal of Multivariate Analysis, Vol. 54, No. 2, pp. 175-192, 1995.

[4] J. W. Silverstein, Strong Convergence of the Empirical Distribution of Eigenvalues of Large Dimensional Random Matrices, Journal of Multivariate Analysis, Vol. 5, No. 11, pp. 331-339, 1995.

[5] V. Morgenshtern and H. Bölcskei, Capacity of Large Amplify-andForward Relay Networks, 2006 IEEE Communication Theory Workshop, Puerto Rico, May 2006.

[6] V. Morgenshtern and H. Bölcskei, Crystallization in Large Wireless Networks, submitted to the IEEE Transactions on Information Theory.

[7] V. Morgenshtern and H. Bölcskei, Large Random Matrix Analysis of Relay Networks, Proceedings of the 2006 Allerton Conference.

[8] S. P. Borade, L. Zheng, and R. G. Gallager, Maximizing Degrees of Freedom in Wireless Networks, Allerton Annual Conference on Communication, Control and Computing, October 2003, pp. 561-570.

[9] I. E. Telatar, Capacity of Multi-Antenna Gaussian Channels, European Transactions on Telecommunications, vol. 10, no. 6, pp. 585-595, 1999. 